\title{
Preservation of Iodised Hydnocarpus Esters
}

Recent experience has shown that iodised hydnocarpus esters may become irritant on prolonged exposure to air and that this process is probably accelerated in the presence of water.

The stock of esters should, therefore, be kept in nearly full containers to reduce contact with the air as much as possible. Where iodised esters have to be imported in bulk, the contents of each large container might be transferred locally into glass bottles large enough to provide, say, not more than one month's supply. The glass bottles used should be sterilised by dry heat and should be thoroughly dry when the drug is transferred. The bottles should be kept well-corked and in a cool, dark place.

If iodised hydnocarpus esters are sterilised by steam before injection they become wet. There is no objection to this so far as immediate injection is concerned, but only the quantity necessary for immediate use should be so treated, and any steam-wetted residues should not be returned to stock bottles. Similarly, wet syringes should not be used to withdraw iodised esters from the stock bottles.

We have been asked by the Wellcome Chemical Research Laboratories to draw the attention of our readers to this matter, and we are very glad to do so.-Editor. 